\title{
Pneumoperitoneum, Pneumothorax, and Pneumoretroperitoneum Post Colonoscopy: A Case report and Review of Literature
}

Authors:

Disclosure:

Ethical approval statement:

Received:

Accepted:

Keywords:

Citation:
Melissa Kyriakos Saad, ${ }^{1}$ Toufic Saber, ${ }^{1}$ George Cortas,, ${ }^{2}$ Elias Saikaly ${ }^{3}$

1. Saint George Hospital University Medical Center, General Surgery Department, University of Balamand, Beirut, Lebanon

2. Gastroenterology Department, Saint George Hospital University Medical Center, University of Balamand, Beirut, Lebanon

3. General Surgery Department, Saint George Hospital University Medical Center, University of Balamand, Beirut, Lebanon

*Correspondence to dreliassaikaly@gmail.com

The authors have declared no conflicts of interest.

Written informed consent was obstained from the patient to publish the case report as well as any associated images.

21.05.20

30.09 .20

Colonoscopy, conservative management, perforation, pneumoperitoneum.

EMJ Gastroenterol. 2020;9[1]:102-105.

\section{Abstract}

Colonic perforation post colonoscopy is rarely seen; however, when coupled with massive pneumoperitoneum in haemodynamically stable patients, a real dilemma for surgeons is created. The decision between watchful waiting versus surgical intervention is the real challenge and while most surgeons will urge for surgical intervention, conservative management on the other hand can be safely applied in selected haemodynamically stable patients.

\section{INTRODUCTION}

Colonoscopy is a common and safe procedure commonly used in clinical practice for the investigation and treatment of a multitude of gastrointestinal pathologies, including both benign and malignant conditions.' Although rarely seen, this procedure is associated with a risk up to $0.3 \%$ of serious complications, such as colonic perforation and bleeding. ${ }^{2,3}$ This risk is seen mainly when a therapeutic approach is used during the procedure. ${ }^{4}$ Pneumoperitoneum is seen in more than $90 \%$ of micro or macrocolonic perforation, and it is defined as free air within the peritoneal cavity. The management dilemma is when the pneumoperitoneum is asymptomatic without signs of peritonitis. Traditionally, antibiotics and surgical management are opted for as first choice in the management of asymptomatic pneumoperitoneum. ${ }^{3,5}$ However, conservative management is becoming more common in such complication, especially in haemodynamically stable patients with nonsurgical abdomen. Here, the authors present a case of benign massive pneumoperitoneum associated with retro-pneumoperitoneum, pneumothorax, and subcutaneous emphysema, diagnosed 2 weeks post diagnostic colonoscopy. 


\section{CASE REPORT}

This is a case of a 64-year-old Caucasian male with comorbid conditions of coronary artery disease, dyslipidaemia, hypertension, and atrial fibrillation who was transferred to the authors institute 2 weeks post screening colonoscopy with severe abdominal distension. At the time of colonoscopy, no risk factors for perforation were documented and no technical challenges were encountered.
During the physical exam, the patient's vitals were stable, with soft but distended abdomen with hypoactive bowel sound and no signs of peritoneal irritation. In addition, decreased bilateral air entry was noted over the lung field on auscultation.

Chest $X$ ray showed pneumothorax and pneumoperitoneum (Figure 1). Kidney, ureter, and bladder $X$ ray (KUB) examination was done in a supine and erected position and showed bowel loop of normal calibres with a large pneumoperitoneum and air fluid levels (Figure 2).

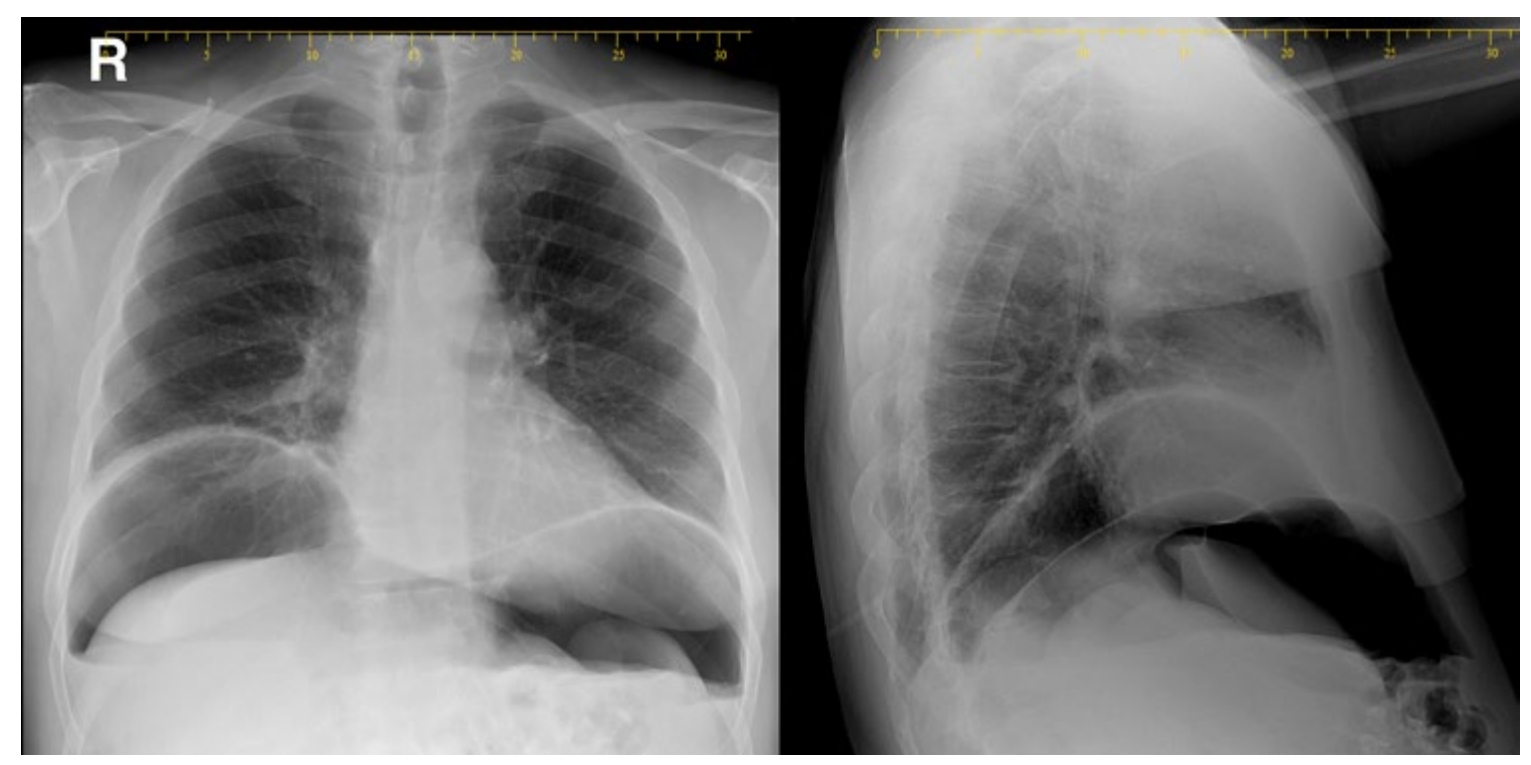

Figure 1: Pneumothorax and pneumoperitoneum.

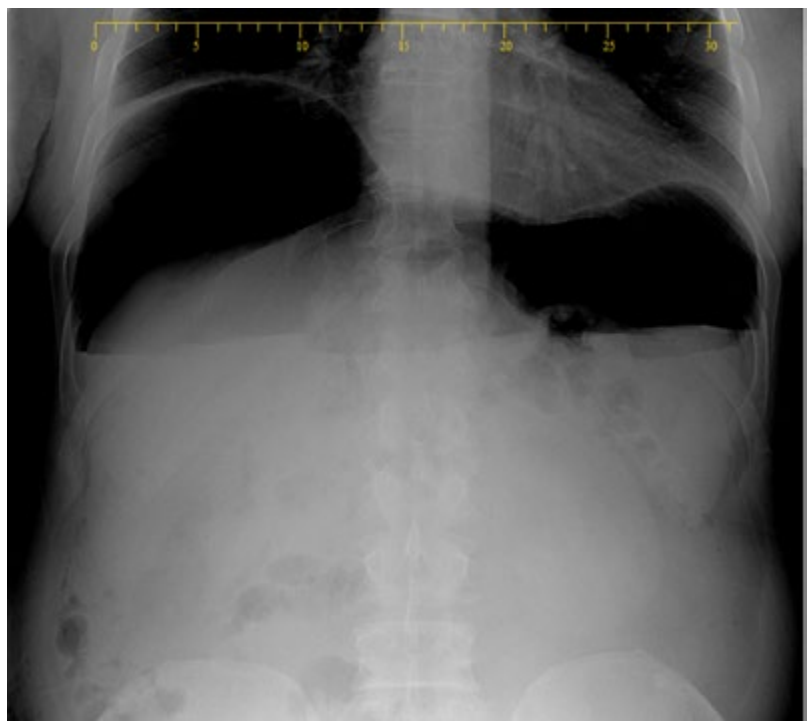

Figure 2: Bowel loop of normal calibres with a large pneumoperitoneum and air fluid levels. 


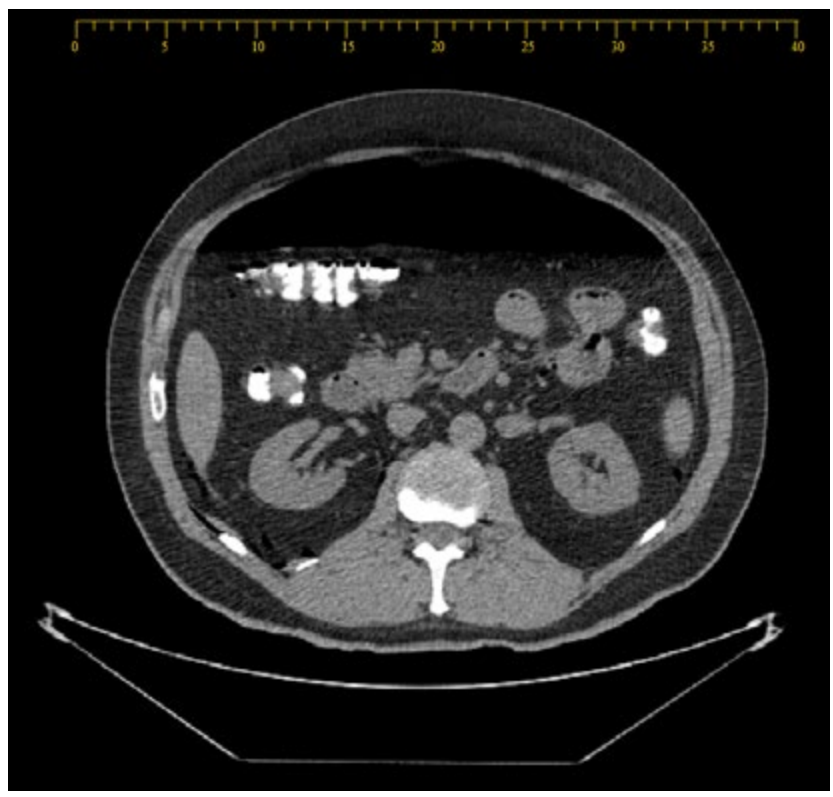

Figure 3: Large pneumoperitoneum in the anterior aspect of the abdomen.

Laboratory tests revealed elevated white blood cell count and C-reactive protein of $16.0 \times 10^{3}$ cells/L and $26.3 \mathrm{mg} / \mathrm{L}$, respectively.

The patient underwent a chest, abdomen, and superior pelvis CT scan, which revealed large pneumoperitoneum in the anterior aspect of the abdomen, free air in the mesentery close to the spleen and liver, free air close to the lesser curvature of the stomach, a thickened sigmoid, thickening in distal ileal loops with fat streaking, pneumoretroperitoneum, and subcutaneous emphysema. The chest CT scan showed pneumothorax and pneumomediastinum and findings were suggestive of a sigmoid perforation (Figure 3).

The patient was haemodynamically stable and afebrile. The decision was taken for a conservative management backboned by keeping the patient nil by mouth; serial repetition of vitals, physical exam, lab tests, $\mathrm{KUB}$; and intravenous antibiotics (amoxiclav, metronidazole and ciprofloxacin) for a total of 7 days, this regimen was started abroad and continued at the authors institute.

Over the next 3 days, the patient's abdominal distension was improving mildly, and his inflammatory markers (white blood cell, neutrophils, and C-reactive protein) improved towards normalisation. On the other hand, his pneumoperitoneum and pneumothorax were relatively stable over the course of his hospitalisation. After 4 days of hospitalisation, the patient developed low-grade fever of (38.5 ${ }^{\circ} \mathrm{C}$ ), while the patient's abdominal physical exam was normal except for distention. This latter fact posed a real dilemma on the origin of the fever, which could be due to lung origin, but any other abdominal cause cannot be ruled out without the diagnostic laparoscopy. Thus, a diagnostic laparoscopy was scheduled aiming to explore the abdominal cavity and at the same time decompress the retained pneumoperitoneum, which would eventually help re-establish the pressure equilibrium between the thoracic and abdominal cavity.

The abdomen was assessed using a $10 \mathrm{~mm}$ under-vision trocar then two $5 \mathrm{~mm}$ trocars were inserted under direct vision. Perihepatic fluids were identified and then sent to culture and cytology, results for which came back negative for malignancies or bacteraemia. No purulent or faecal materials were detected. Severe inflammatory reaction was noted at the level of the sigmoid with loops of small bowel adherent to the sigmoid, in favour of a contained or walled off sigmoid perforation. The decision was made to drain the abdominal cavity using a 24 French gauge Blake drain placed in the pelvis and near the presumed sigmoid perforation. 
On postoperative Day 2, the patient started a full liquid diet, which was tolerated and advances in diet were not made until soft gastric low residue was reached on Day 4 post operation. The patient was discharged home on Day 6 post diagnostic laparoscopy, without antibiotics. The patient opted to repeat his colonoscopy in 6-8 weeks time, as he was staying in the country for medical care only, if his physical exam and followup laboratory workup were normal, and at 3 months if any abnormality was noted.

\section{DISCUSSION}

The authors review of literature revealed only a few reported cases of massive pneumoperitoneum secondary to colonic perforation from colonoscopy that were managed conservatively. Even rarer, is the delayed presentation of patients with post colonoscopic perforation 2 weeks after colonoscopy. In fact, the frequency of perforations due to colonoscopy ranges from as low as $0.2 \%$ when diagnostic purpose is targeted and can reach as high as $2.0 \%$ when aiming for therapeutic intent. 3,6 In fact, $9 \%$ of the colonoscopic perforations were identified at least 2 weeks after the procedure. In this case, colonic perforation was detected after 14 days. Colonic perforation when coupled with massive pneumoperitoneum in haemodynamically stable patients creates a real dilemma for surgeons nowadays. The decision between watchful waiting versus surgical intervention is the real challenge. While most surgeons will urge for surgical intervention, conservative management on the other hand can be safely applied in selected haemodynamically stable patients. Thus, conservative treatment for massive pneumoperitoneum is suitable based on the patient's symptoms and clinical condition. If the abdominal pain was mild and localised and no severe sepsis or peritonitis was perceived, watchful waiting is adequate. ${ }^{5}$ Henceforth, the decision for the trial of a conservative management in the authors' case was made: the patient presented with no signs of peritonitis and was haemodynamically stable.

The picture of a massive pneumoperitoneum can be shocking at the time of diagnosis but understanding the pathophysiology of its occurrence supports the decision of nonsurgical management. In the cases of a viscus perforation enteric contamination occurs and only a small amount of air escapes. ${ }^{6}$ However, the picture of massive pneumoperitoneum is mainly encountered in nonsurgical cases since no signs of peritonitis or sepsis are present and consequently more air enters the peritoneal cavity. ${ }^{6}$

To the authors' knowledge, no defined algorithm exists at the moment for the management of these clinical scenarios. But what is undebatable is that the most significant aspect that the physician should base their decision upon is the general condition of the patient. Indeed, conservative management is successful in cases of massive pneumoperitoneum, and close monitoring of the patient's vitals and serial abdominal physical exam are enough. Alarming signs such as peaks of fever and increased intensity of abdominal pain shall urge the surgeon to reconsider their decision for a conservative management.

\section{CONCLUSION}

In conclusion, management of massive pneumoperitoneum is always challenging in haemodynamically stable patients and treatment should consider a balance between conservative management versus surgical intervention.

\section{References}

1. Senore C et al. (2016). Colonoscopy surveillance: guidelines for polyps and IBD. Minerva Gastroenterol Dietol. 2016;62(2):207-22

2. Bharadwaj $S$ et al. The role of endoscopy in inflammatory bowel disease. J Dig Dis. 2015;16(12):689-98.

3. Alexiou $\mathrm{K}$ et al. Subcutaneous emphysema, pneumomediastinum and pneumoperitoneum after unsuccessful ERCP: a case report. Cases J. 2009;2(1):120.

4. Marwan $\mathrm{K}$ et al. Pneumothorax, pneumomediastinum, pneumoperitoneum, pneumoretroperitoneum and subcutaneous emphysema following diagnostic colonoscopy. Ann R Coll
Surg Engl. 2007;89(5):W20-1.

5. Ustek $\mathrm{S}$ et al. Benign pneumoperitoneum after colonoscopy. Case Rep Med. 2010;2010:631036.

6. Wang H, Batra V. Massive pneumoperitoneum presenting as an incidental finding. Cureus. 2018;10(6):e2787. 